\title{
Enhanced localized fluorescence in plasmonic nanoantennae
}

Bakker, R.M.; Yuan, H.-K.; Liu, Z.; Drachev, V.P.; Kildishev, A.V.; Shalaev, V.M.; Pedersen, Rasmus Haugstrup; Gresillon, S.; Boltasseva, Alexandra

Published in:

Applied Physics Letters

Link to article, DOI:

$10.1063 / 1.2836271$

Publication date:

2008

Document Version

Publisher's PDF, also known as Version of record

Link back to DTU Orbit

Citation (APA):

Bakker, R. M., Yuan, H-K., Liu, Z., Drachev, V. P., Kildishev, A. V., Shalaev, V. M., Pedersen, R. H., Gresillon, S., \& Boltasseva, A. (2008). Enhanced localized fluorescence in plasmonic nanoantennae. Applied Physics Letters, 92(4), 043101. https://doi.org/10.1063/1.2836271

\section{General rights}

Copyright and moral rights for the publications made accessible in the public portal are retained by the authors and/or other copyright owners and it is a condition of accessing publications that users recognise and abide by the legal requirements associated with these rights.

- Users may download and print one copy of any publication from the public portal for the purpose of private study or research.

- You may not further distribute the material or use it for any profit-making activity or commercial gain

- You may freely distribute the URL identifying the publication in the public portal 


\title{
Enhanced localized fluorescence in plasmonic nanoantennae
}

\author{
Reuben M. Bakker, ${ }^{\text {a) }}$ Hsiao-Kuan Yuan, Zhengtong Liu, Vladimir P. Drachev, \\ Alexander V. Kildishev, and Vladimir M. Shalaev \\ School of ECE, Birck Nanotechnology Center, Purdue University, W. Lafayette, Indiana 47907, USA \\ Rasmus H. Pedersen \\ MIC-Department of Micro and Nanotechnology, Technical University of Denmark (DTU), DK-2800 Kgs \\ Lyngby, Denmark \\ Samuel Gresillon \\ LPEM UPRA5, CNRS and Université P. et M. Curie Paris 6, F-75005 Paris, France
}

Alexandra Boltasseva

COM.DTU, Technical University of Denmark (DTU), DK-2800 Kgs Lyngby, Denmark

(Received 17 October 2007; accepted 24 December 2007; published online 28 January 2008)

\begin{abstract}
Pairs of gold elliptical nanoparticles form antennae, resonant in the visible. A dye, embedded in a dielectric host, coats the antennae; its emission excites plasmon resonances in the antennae and is enhanced. Far-field excitation of the dye-nanoantenna system shows a wavelength-dependent increase in fluorescence that reaches 100 times enhancement. Near-field excitation shows enhanced fluorescence from a single nanoantenna localized in a subwavelength area of $\sim 0.15 \mu \mathrm{m}^{2}$. The polarization of enhanced emission is along the main antenna axis. These observed experimental results are important for increasing light extraction from emitters localized around antennae and for potential development of a subwavelength sized laser. (C) 2008 American Institute of Physics.

[DOI: $10.1063 / 1.2836271]$
\end{abstract}

Localized surface plasmon resonances (LSPRs) are collective oscillations of electrons in metal nanoparticles driven by electromagnetic radiation ${ }^{1}$ (see also a recent review ${ }^{2}$ ). This resonance is a function of metal and host dielectric functions, particle geometry, along with light polarization and wavelength. LSPRs can generate high, localized electromagnetic fields which entice scientists and engineers for a variety of achieved and future applications. These include Raman scattering, ${ }^{3,4}$ single molecule fluorescence, ${ }^{5,6}$ enhanced emission from dyes and quantum dots (QDs), ${ }^{7-15}$ various nonlinear optical processes, ${ }^{16-18}$ and the basis for a subwavelength sized nanolaser. ${ }^{19-21}$

The nanoantenna (also known as an optical antenna) system consisting of single or coupled nanoparticles, either isolated or arrayed, is well studied. ${ }^{5-15,22-29}$ The system of two closely spaced nanoparticles is of particular interest as the LSPR of individual particles couple, resulting in greater electric field enhancement ${ }^{24-26}$ and a systematic red shift compared to a single particle. ${ }^{22-24,28}$ Field enhancements afforded by LSPRs result in enhanced emission of dyes and QDs in the vicinity of the nanoparticles. ${ }^{5-15}$ Briefly, the source of the enhancement is twofold: feedback between the emitter and the resonant nanoparticles increases the emitter's radiative decay rate so the photon emission rate increases and the nanoantenna system acts as a transducer for light from the near field to the far field. ${ }^{8-13,15}$ Studying the interactions of a nanoantenna with localized, nanosized emitters makes a strong starting point for the development of LSPR-based lighting devices with properties such as high spatial localization of a usable light source and efficiency improvements for light extraction from emitters, including QDs and carbon nanotubes (CNTs) ${ }^{5-15,27,30}$ It is postulated that laserlike light

${ }^{a)}$ Electronic mail: rbakker@purdue.edu. can coherently emit from a nanoantenna system coupled to a localized gain medium. Such a nanolaser is theoretically possible if the gain factor from the emitter can overcome losses and coherent, near-field feedback is established between the nanoantenna and the gain medium. ${ }^{19-21}$

We report on nanoantenna-based LSPRs excited by emission of a fluorescent dye embedded around both isolated and arrayed antenna structures and the observation of enhanced fluorescence and localized emission from a subwavelength sized device. The dye is excited at a wavelength away from the LSPR, but its emission overlaps with and drives the plasmon resonance. Far-field spectral measurements show 20-100 times enhanced fluorescence for the investigated wavelength range. Near-field scanning optical microscope (NSOM) illumination characterizes the local nature of the enhancement. It shows wavelength-integrated enhancements over 30 and 7 times for arrayed and single antenna structures. Enhanced emission from an isolated device is contained to a subwavelength area of $0.15 \mu \mathrm{m}^{2}$. Compared to previous demonstrations of enhanced fluorescence, the presented results combine demonstration of a high enhancement factor with near-field measurements showing localization of enhanced emission from a single nanoantenna and the polarization of enhanced light, all from a well defined nanoantenna design.

The nanoantenna system used in this study consists of two gold nanoparticles. The particles (elliptical cylinders) are separated by a small gap along their major axes as seen in Fig. 1 and are similar in design to those reported in Ref. 29. The pattern is written in a resist (ZEP520A) on a quartz substrate using electron beam lithography (JEOL JBX9300FS). After writing, $40 \mathrm{~nm}$ of gold is deposited with an electron beam evaporator and a liftoff technique leaves high quality pairs in a well defined array; see field emission scanning electron microscope (FESEM) images in Figs. 1(c) and 


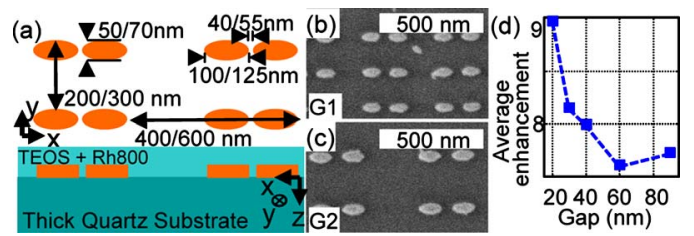

FIG. 1. (Color online) Nanoantenna array: (a) top and side sketch of the sample. (b) FESEM image of geometry 1; small dimensions. (c) FESEM image of geometry 2; large dimensions. (d) Simulated volume and wavelength averaged field enhancements over unit cell volume where dye would be located for a structure similar to geometry 1 .

1(d). To study the effect of a randomly localized emitter on a nanoantenna (and vice versa), a fluorescent dye embedded in a dielectric is coated around the antennae. Rhodamine 800 (Rh800) is incorporated into a glass matrix by sol-gel processing. Tetraethoxysilane, ethanol, water, and $0.1 \mathrm{ml}$ hydrochloric acid (added as a catalyst) are mixed at a molar ratio of $1: 4: 3$ for $3 \mathrm{~h}$. Rh800 in powder form is mixed into the solution for $8 \mathrm{~h}$ at room temperature. The dye-doped solution is spin coated on the sample and baked at $60{ }^{\circ} \mathrm{C}$ for another $8 \mathrm{~h}$. The dielectric layer is back etched to a thickness of $85 \mathrm{~nm}$ using reactive ion etching (PlasmaLab). This reduces the volume of Rh800 not interacting with the antennae and allows for NSOM excitation of the nanoantenna-dye system. The concentration of Rh800 is $4.8 \mathrm{mMol} / \mathrm{L}$. Figure 1(d) shows a volume and wavelength averaged electric field enhancement as a function of gap size for a structure similar to geometry 1 . The enhancement over a unit cell volume with a thickness of $80 \mathrm{~nm}$ shows that field enhancements increase with decreasing gap size.

Far-field spectroscopy is used to characterize the transmission and reflection spectra of the antenna arrays (SpectraCode collection system). For x-polarized light, the two presented geometries exhibit a strong electrical resonance in the red portion of the spectrum. It is seen as an increase in reflection from the sample and is illustrated in Fig. 2(a). This resonance originates from the ellipses' major axis length $(X$ direction). A smaller coupling distance between adjacent particles strengthens and redshifts the resonance. For the orthogonal polarization, coupled particles exhibit a weaker resonance at shorter wavelengths (in the green) due to their minor axis length ( $Y$ direction). ${ }^{29}$

The fluorescence spectrum of Rh800 overlaps with the LSPR of the two presented antenna geometries; see Fig. 2. Given this overlap, the Stokes shift of Rh800 emission from excitation at $633 \mathrm{~nm}$ and the LSPR wavelength range, Rh800

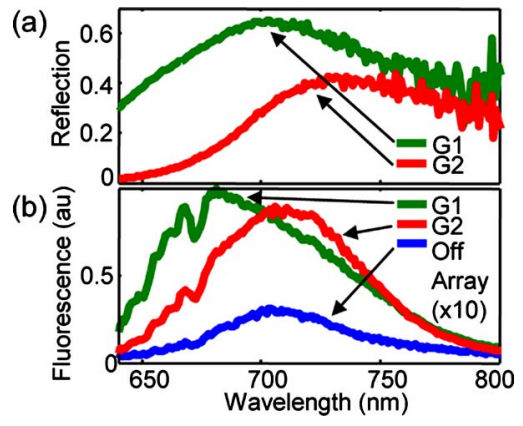

FIG. 2. (Color online) Far-field characterization: (a) broadband spectral response for x-polarized light. (b) Antenna enhanced fluorescence of Rh800 compared with Rh800 fluorescence away from the antennae (633 nm excitation). The dip at $680 \mathrm{~nm}$ is from a long pass filter in the collection path.

Downloaded 21 Jun 2010 to 192.38.67.112. Redistribution subject
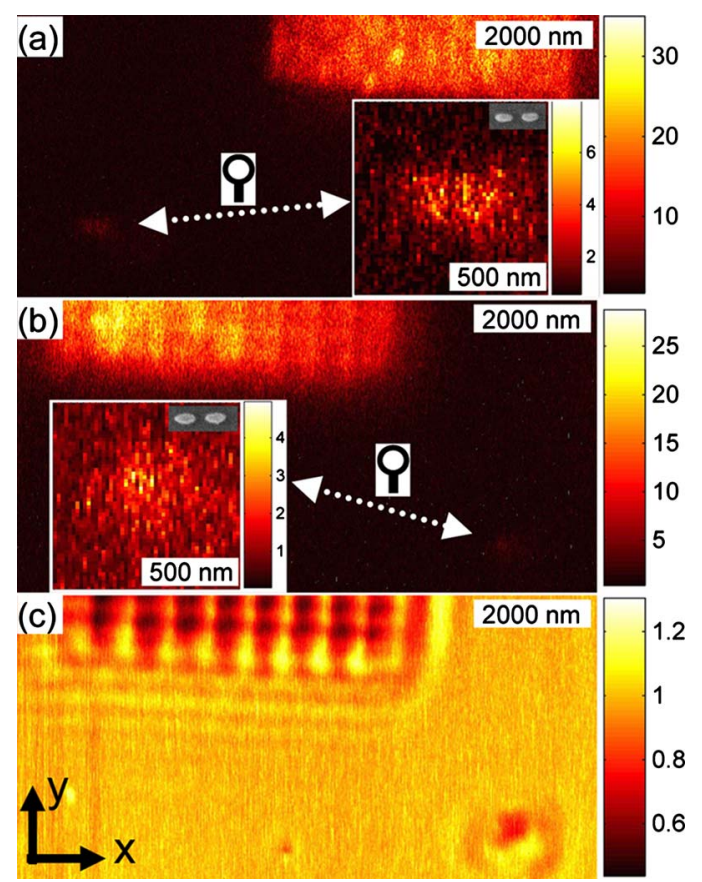

FIG. 3. (Color online) Illumination mode NSOM: (a) near-field integrated fluorescence for geometry 1 showing localized enhancements of over 30 times for arrayed and seven times for isolated antennae. (b) Same as (a) but for geometry 2 showing localized enhancement over 25 for arrayed and 4 for isolated antennae. (c) Near-field image of the same area as (b) at the excitation wavelength plus the fluorescence signal. Insets: zoomed in view of an isolated antenna with FESEM of antenna shown to scale. Color scale: 1 is the "background" value, away from any antenna. Collected light is not polarization specific.

is an excellent candidate to characterize the system of a dye exciting a nanoantenna structure with minimal interaction from the original $633 \mathrm{~nm}$ excitation. To investigate the spectral dependence of emission from the Rh800, nanoantenna system, far-field radiation excites the dye and backscattered emission is detected (with a Renishaw inVia spectrometer). Figure 2(b) shows enhanced fluorescence compared with Rh800 fluorescence away from the nanoantenna arrays. Intensity measurements taken from Rh800 embedded around the nanoantenna structures $\left(I_{\mathrm{EF}}(\lambda)\right)$ show significant enhancement compared with the signal away from the nanoantennae $\left(I_{F}(\lambda)\right)$. The enhancement $\left(I_{\mathrm{EF}}(\lambda) / I_{F}(\lambda)\right)$ ranges from 20 to 100 as a function of wavelength. Spectrally, the LSPR has a strong effect in enhancing the fluorescence; the two presented geometries show different enhanced fluorescence spectra which are inline with their LSPRs.

To obtain a spatially resolved picture of antenna-induced fluorescence enhancement, an aperture-based NSOM (Nanonics) excites the sample with $633 \mathrm{~nm}$ light in illumination mode and backscattered light is collected. The NSOM uses atomic force feedback to hold an aperture in the nearfield region of the sample while the sample is raster scanned beneath. The aperture, with an estimated diameter of $200 \mathrm{~nm}$, is drawn from a single mode fiber and delivers linearly polarized light to the sample (with an estimated 10:1 extinction ratio). Collected light passes through a $650 \mathrm{~nm}$ long pass filter (at least $\mathrm{OD}=7.0$ at $633 \mathrm{~nm}$ ) to remove the excitation wavelength and the integrated fluorescence signal is measured with an avalanche photodiode. For comparison, data are collected without the color filter to see the effects of the pump wavelength on the antenna array. All NSOM reo AIP license or copyright; see http://apl.aip.org/apl/copyright.jsp 


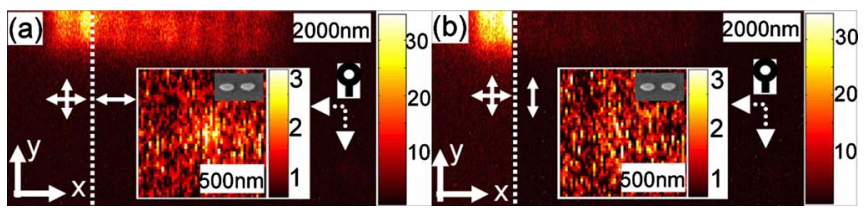

FIG. 4. (Color online) Characterizing the polarization of emitted light for geometry 2: (a) near-field integrated fluorescence; at the dotted line, the collected light changes from all emitted light to only x-polarized light (solid arrows indicate polarization of collected light). (b) Same as (a) but from all emission to only y-polarized light. Insets: zoomed in view of the isolated antenna with FESEM of antenna shown to scale. Color scale: 1 is the fluorescence signal, away from any antenna. These measurements are from the same area presented in Figs. 3(b) and 3(c).

sults are for excitation light polarized parallel to the major antenna axis.

Figure 3 shows a comparison of enhanced fluorescence for geometries 1(a) and 2(b) along with NSOM measurements at the excitation wavelength for geometry 2(c). The measurements include an isolated particle pair and arrayed antennae. Figure 3 shows a localized increase in fluorescence intensity by over 30 times for geometry 1 and 25 times for geometry 2 compared to the weak fluorescence signal away from the antenna structures. For the isolated pairs, geometry 1 (40 nm gap) shows emission contained to a subwavelength area of $500 \times 300 \mathrm{~nm}^{2}$ with an enhancement factor of 7 , while geometry 2 (55 nm gap) shows an area of 500 $\times 500 \mathrm{~nm}^{2}$ with an enhancement factor of 4 . The NSOM image collected with no color filter in the collection path has a signal over 4000 times stronger [not shown as Fig. 3(c) is normalized] than the enhanced fluorescence signal and shows the effects of the excitation wavelength on the sample. Figure 3(c) shows scattering features from both arrayed and isolated antennae. These features do not represent resonant scattering from a LSPR, as the contrast ratio between $I_{\max }$ and $I_{\min }$ is very low compared with NSOM imaging on a nanoantenna sample at a resonant wavelength. ${ }^{29}$

A linear polarizer placed in the collection path is used to observe the polarization state of the enhanced fluorescence and is characterized to be mainly parallel to the major antenna axis. With x-polarized light [Fig. 4(a)], up to $\sim 70 \%$ of the enhanced fluorescence signal reaches the detector from the array and the isolated pair also shows enhanced fluorescence. For y-polarized light [Fig. 4(b)], the signal over the array is reduced, with only $\sim 15 \%$ of the enhanced fluorescence reaching the detector and the isolated nanoantenna shows no fluorescence signal above the background.

Emission from an embedded dye is shown to excite a LSPR in well defined nanoantennae. This results in an increase in fluorescence from 20 to 100 times as a function of wavelength. Illumination-mode NSOM shows that enhanced emission can be localized to an area of $\sim 0.15 \mu \mathrm{m}^{2}$ for an isolated antenna and the polarization of the emitted light is parallel to the major antenna axis. The presented work should prove useful for future light-emitting devices requiring high light extraction from dyes, QDs, CNTs, and other emitters in addition to the development of a LSPR-based nanolaser.

The authors acknowledge partial support from AROSTTR under Award No. W911NF-07-C-0008, ARO MURI award number 50432-PH-MUR and NSF-PREM award No. DMR-0611430, and the Danish Research Agency NABIIT program [Grant No. 2106-05-0033 (AB)], and CNRS (SG, AVK).

${ }^{1}$ U. Kreibig and M. Vollmer, Optical Properties of Metal Clusters (Springer, Berlin, 1995).

${ }^{2}$ E. Ozbay, Science 311, 189 (2006).

${ }^{3}$ M. Moskovits, J. Raman Spectrosc. 36, 485 (2005).

${ }^{4}$ E. Y. Poliakov, V. M. Shalaev, V. A. Markel, and R. Botet, Opt. Lett. 21, 1628 (1996).

${ }^{5}$ P. Anger, P. Bharadwaj, and L. Novotny, Phys. Rev. Lett. 96, 113002 (2006).

${ }^{6}$ S. Kuhn, U. Hakanson, L. Rogobete, and V. Sandoghdar, Phys. Rev. Lett. 97, 017402 (2006).

${ }^{7}$ H. Diltbacher, J. R. Krenn, N. Felidj, B. Lamprecht, G. Schider, M. Salerno, A. Leitner, and F. R. Aussenegg, Appl. Phys. Lett. 80, 404 (2002).

${ }^{8}$ J.-H. Song, T. Atay, S. Shi, H. Urabe, and A. V. Nurmikko, Nano Lett. 5, 1557 (2005).

${ }^{9}$ J. N. Farahani, D. W. Pohl, H.-J. Eisler, and B. Hecht, Phys. Rev. Lett. 95, 017402 (2005).

${ }^{10}$ T. D. Corrigan, S.-H. Guo, R. J. Phaneuf, and H. Szmacinski, J. Fluoresc. 15, 777 (2005).

${ }^{11}$ J. S. Biteen, N. S. Lewis, H. A. Atwater, H. Mertens, and A. Polman, Appl. Phys. Lett. 88, 131109 (2006).

${ }^{12}$ H. Mertens and A. Polman, Appl. Phys. Lett. 89, 211107 (2006).

${ }^{13}$ S. Gerber, F. Reil, U. Hohenester, T. Schlagenhaufen, J. R. Krenn, and A. Leitner, Phys. Rev. B 75, 073404 (2007).

${ }^{14}$ Y. Chen, K. Munechika, and D. S. Ginger, Nano Lett. 7, 690 (2007).

${ }^{15}$ O. L. Muskens, V. Giannini, J. A. Sanchez-Gil, and J. Gomez Rivas, Nano Lett. 7, 2871 (2007).

${ }^{16}$ A. M. Dykhne, A. K. Sarychev, and V. M. Shalaev, Phys. Rev. B 67, 195402 (2003).

${ }^{17}$ V. M. Shalaev, R. Botet, and A. V. Butenko, Phys. Rev. B 48, 6662 (1993).

${ }^{18}$ A. V. Butenko, V. M. Shalaev, and M. I. Stockman, Z. Phys. D: At., Mol. Clusters 10, 81 (1988).

${ }^{19}$ D. J. Bergman and M. I. Stockman, Phys. Rev. Lett. 90, 027402 (2003).

${ }^{20}$ I. E. Protsenko, A. V. Uskov, O. A. Zaimidoroga, V. N. Samoilov, and E. P. O'Reilly, Phys. Rev. A 71, 063812 (2005).

${ }^{21}$ A. K. Sarychev and G. Tartakovsky, Phys. Rev. B 75, 085436 (2007).

${ }^{22}$ W. Rechberger, A. Hohenau, A. Leitner, J. R. Krenn, B. Lamprecht, and F. R. Aussenegg, Opt. Commun. 220, 137 (2003).

${ }^{23}$ K. H. Su, Q. H. Wei, X. Zheng, J. J. Mock, D. R. Smith, and S. Schultz, Nano Lett. 3, 1087 (2003).

${ }^{24}$ A. Sundaramurthy, K. B. Crozier, G. S. Kino, D. P. Fromm, P. J. Schuck, and W. E. Moerner, Phys. Rev. B 72, 165409 (2005).

${ }^{25}$ P. Muhlschlegel, H. J. Eisler, O. J. F. Martin, B. Hecht, and D. W. Pohl, Science 308, 1607 (2005).

${ }^{26}$ E. Cubukcu, E. A. Kort, K. B. Crozier, and F. Capasso, Appl. Phys. Lett. 89, 093120 (2006).

${ }^{27}$ L. Rogobete, F. Kaminski, M. Agio, and V. Sandoghdar, Opt. Lett. 32, 1623 (2007).

${ }^{28}$ P. K. Jain, W. Huang, and M. A. El-Sayed, Nano Lett. 7, 2080 (2007).

${ }^{29}$ R. M. Bakker, A. Boltasseva, Z. Liu, R. H. Pedersen, S. Gresillon, A. V. Kildishev, V. P. Drachev, and V. M. Shalaev, Opt. Express 15, 13682 (2007).

${ }^{30}$ J. A. Misewich, R. Martel, Ph. Avouris, J. C. Tsang, S. Heinze, and J. Tersoff, Science 300, 783 (2003). 